Supplement of Geosci. Model Dev., 13, 1137-1153, 2020

https://doi.org/10.5194/gmd-13-1137-2020-supplement

(C) Author(s) 2020. This work is distributed under

the Creative Commons Attribution 4.0 License.

(c) (1)

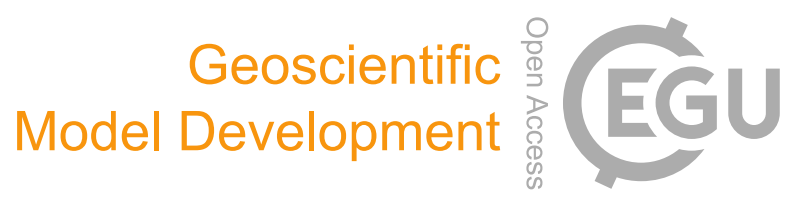

Supplement of

\title{
Implementation of Yale Interactive terrestrial Biosphere model v1.0 into GEOS-Chem v12.0.0: a tool for biosphere-chemistry interactions
}

Yadong Lei et al.

Correspondence to: Xu Yue (yuexu@nuist.edu.cn)

The copyright of individual parts of the supplement might differ from the CC BY 4.0 License. 
Supplementary Information

5

6 Table.S1 The coefficient $a$ of $\mathrm{O}_{3}$-damaging sensitivity for a specific PFT.

\begin{tabular}{lcc}
\hline PFTs & $\begin{array}{c}a \text { for high sensitivity } \\
\left(\mathrm{mmol}^{-1} \mathrm{~m}^{-2}\right)\end{array}$ & $\begin{array}{c}a \text { for low sensitivity } \\
\left(\mathrm{mmol}^{-1} \mathrm{~m}^{-2}\right)\end{array}$ \\
\hline Evergreen broadleaf forest & 0.15 & 0.04 \\
Evergreen needleleaf forest & 0.075 & 0.02 \\
Deciduous broadleaf forest & 0.15 & 0.04 \\
Shrub & 0.1 & 0.03 \\
Tundra & 0.1 & 0.03 \\
$\mathrm{C}_{4}$ grasses & 0.735 & 0.13 \\
$\mathrm{C}_{3}$ grasses & 1.4 & 0.25 \\
$\mathrm{C}_{3}$ crops & 1.4 & 0.25 \\
$\mathrm{C}_{4}$ crops & 0.735 & 0.13 \\
\hline
\end{tabular}

7

8

9

10

11

12 
Table S2. The conversion relationships between YIBs and GEOS-Chem deposition land types.

\begin{tabular}{ll}
\hline YIBs land types & GEOS-Chem deposition land types \\
\hline Evergreen broadleaf forest & Amazon forest \\
Evergreen needleleaf forest & Coniferous forest \\
Deciduous broadleaf forest & Deciduous forest \\
Shrub & Shrub/grassland \\
Tundra & Shrub/grassland \\
$\mathrm{C}_{4}$ grasses & Shrub/grassland \\
$\mathrm{C}_{3}$ grasses & Shrub/grassland \\
$\mathrm{C}_{3}$ crops & Agricultural land \\
$\mathrm{C}_{4}$ crops & Agricultural land \\
\hline
\end{tabular}


(a) Evergreen broadleaf forest

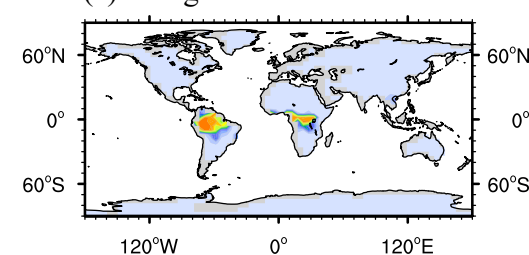

(d) Tundra

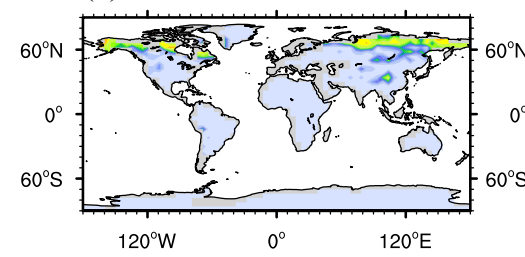

(g) $\mathrm{C}_{3}$ grasses

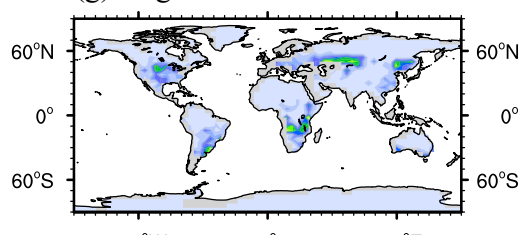

$120^{\circ} \mathrm{W}$ (b) Evergreen needleleaf forest

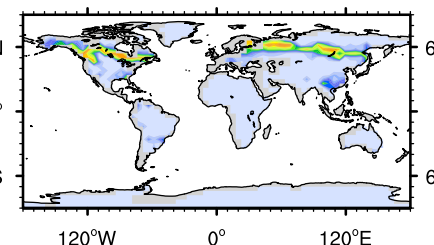

(e) Shrub

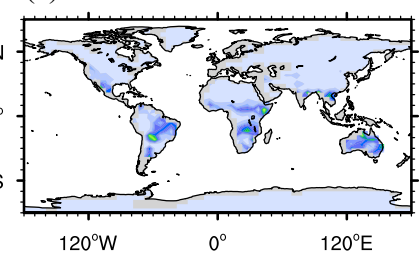

(h) $\mathrm{C}_{4}$ crops

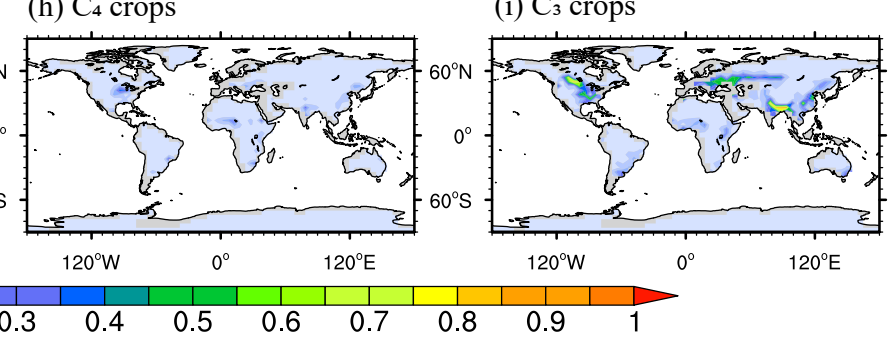

(c) Deciduous broadleaf forest

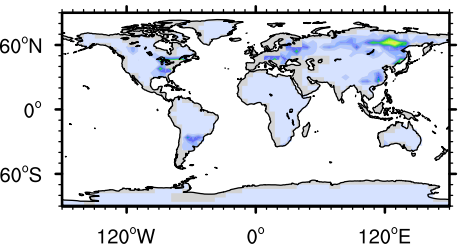

(f) $\mathrm{C}_{4}$ grasses

Figure S1 Fractional coverage of each land type at each grid cell. 


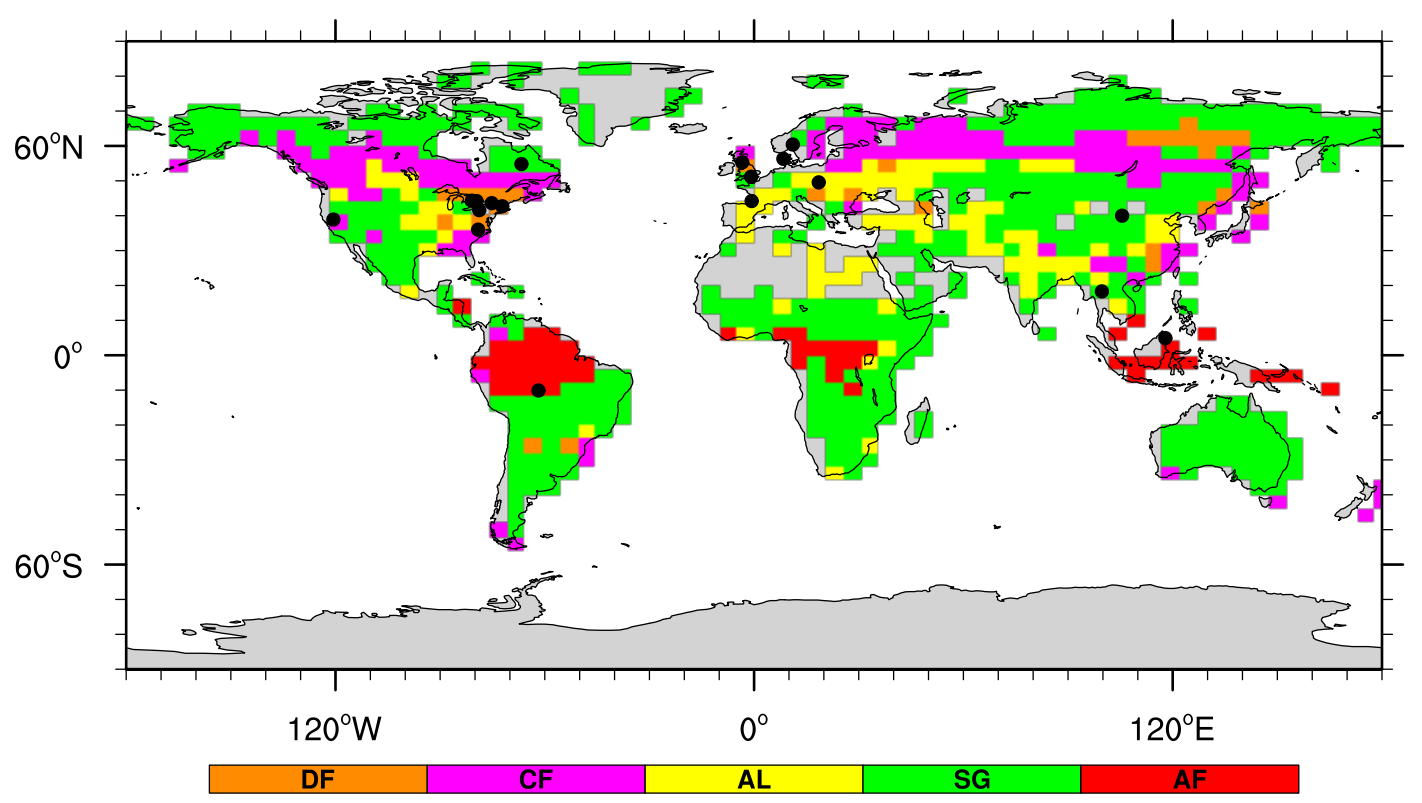

39

40 Figure S2 The major dry deposition land type at each grid cell converted from YIBs land types. DF, CF, AL, SG and AF represent deciduous forest, coniferous forest, agricultural land, shrub/grassland and amazon forest, respectively. Black dots indicate the locations of measurement sites used in evaluation (Table 2).

44

45 
(a) YIBs land types

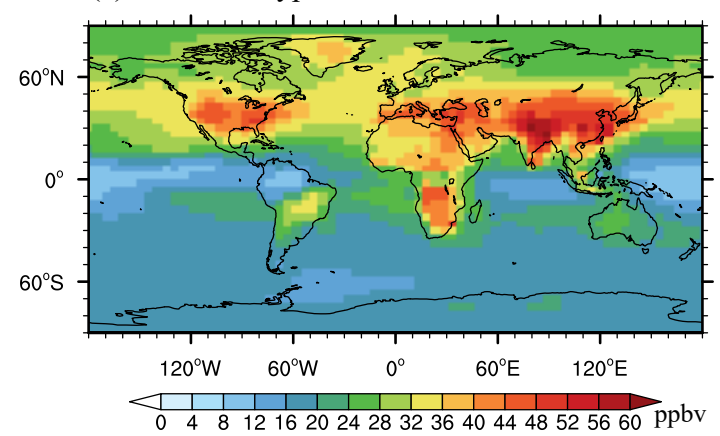

(b) Olson land types

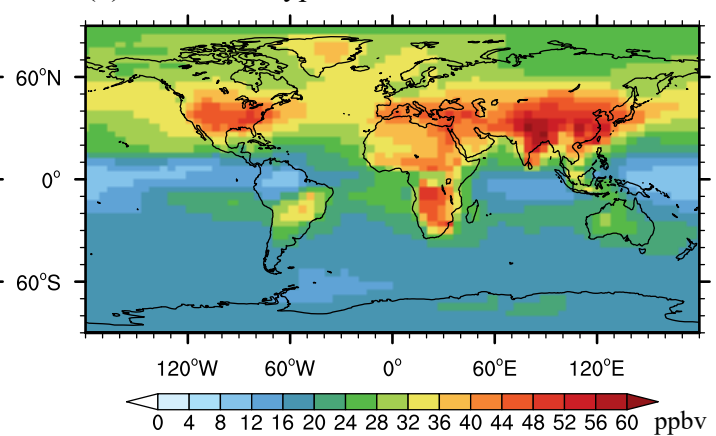

(c) YIBs land types - Olson land types

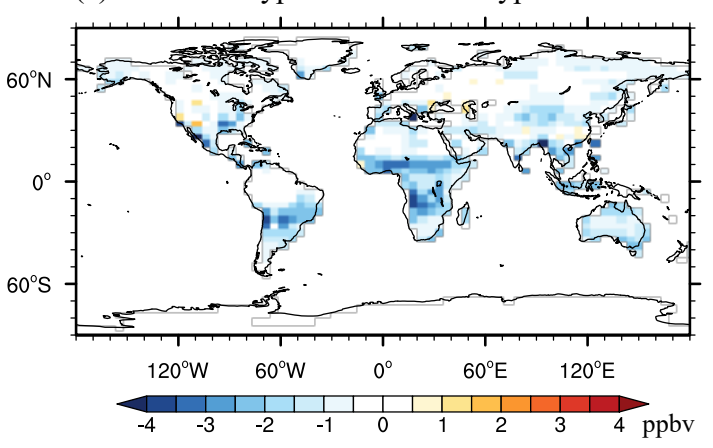

Figure S3 Comparison of YIBs and Olson land types. (a) and (b) represent the simulated MDA8 $\left[\mathrm{O}_{3}\right]$ using YIBs land types and Olson land types, respectively. (c) represents the simulated MDA8 $\left[\mathrm{O}_{3}\right]$ difference between YIBs and Olson land types. (1) 
(a) Simulated MDA8 (51.5 ppbv)

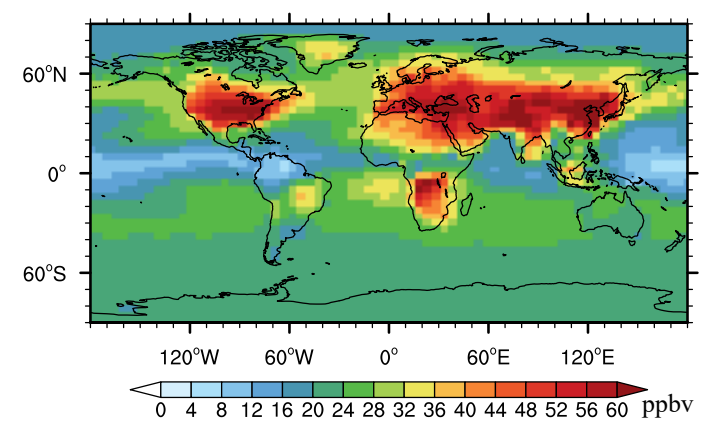

(b) Observed MDA8 (49.2 ppbv)

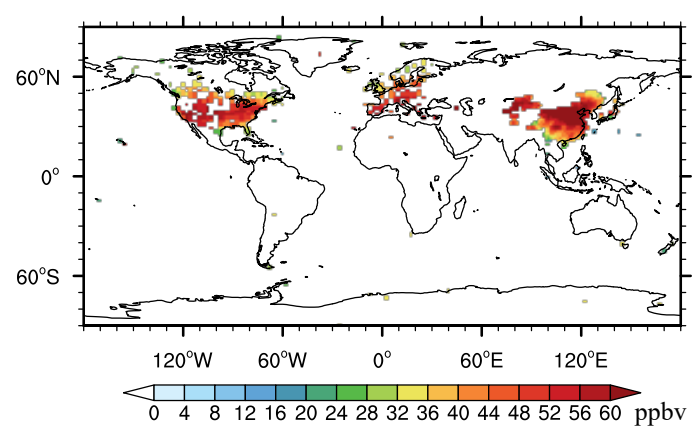

(c) $\triangle$ MDA8 (Model minus observation)

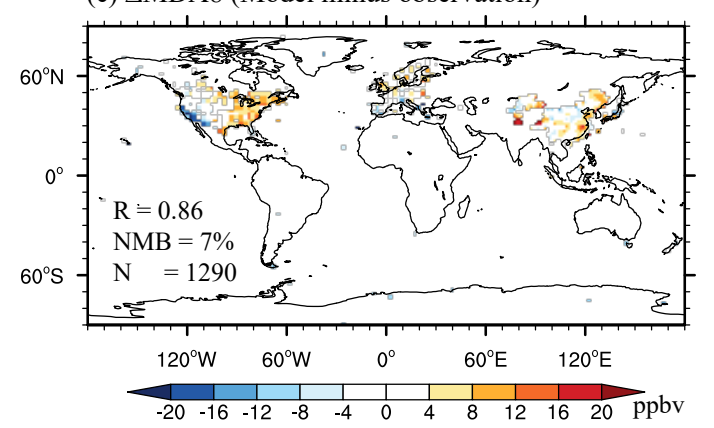

Figure S4 Summertime mean surface $\mathrm{O}_{3}$ concentrations from simulations (a), observations (b), and their differences (c) averaged for period of 2010-2012. Global area-weighted surface $\mathrm{O}_{3}$ concentrations over grids with available observations are shown on the title brackets. The correlation coefficient $(\mathrm{R})$, global normalized mean biases (NMB), and grid numbers $(\mathrm{N})$ with observations are shown in the bottom figure. 
(a) Isoprene

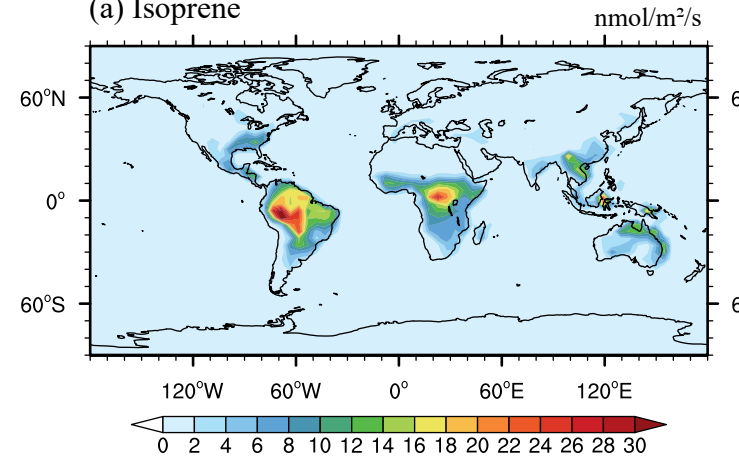

(c) NOx

(b) $\Delta$ Isoprene driven by coupled LAI $\mathrm{nmol} / \mathrm{m}^{2} / \mathrm{s}$

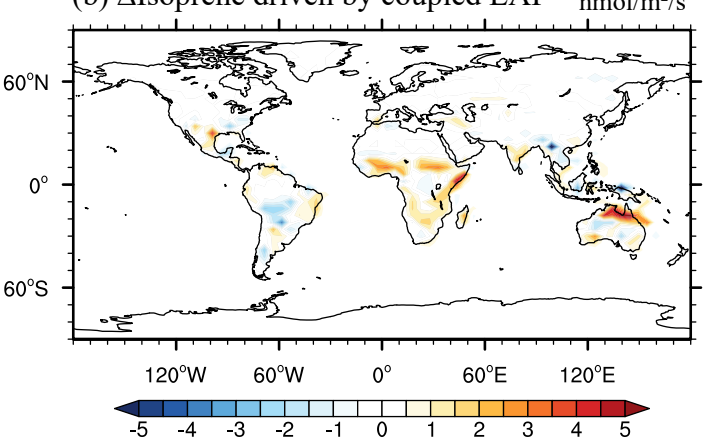

(d) $\triangle$ NOx driven by coupled LAI $10^{\wedge} 10 \mathrm{~mol} / \mathrm{cm}^{2} / \mathrm{s}$
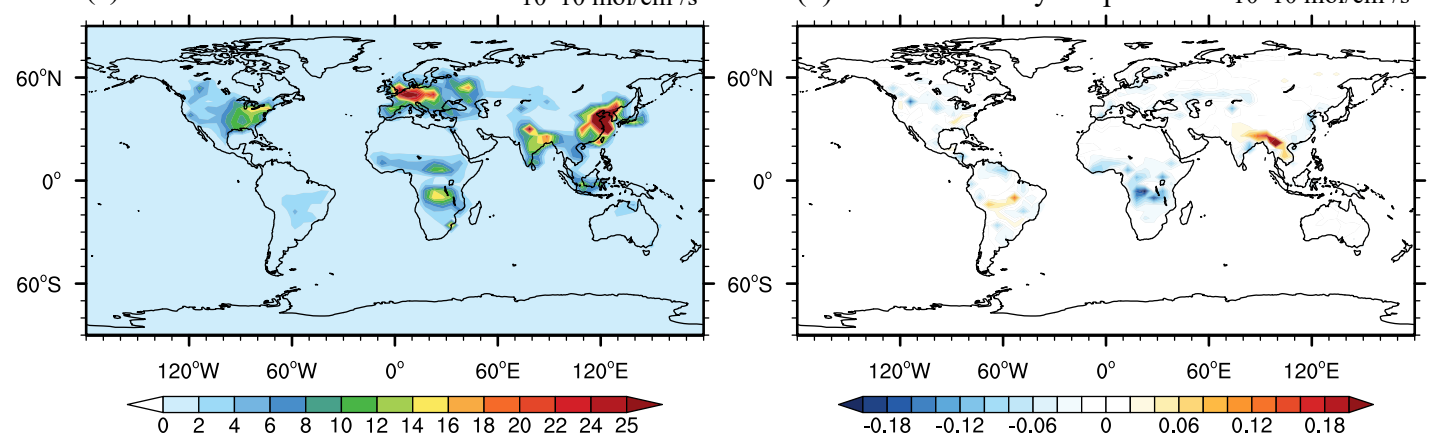

61

Figure S5 Simulated annual isoprene (a) and $\mathrm{NO}_{\mathrm{x}}$ (c) emissions from online GC-YIBs model and its changes (b-d) caused by coupled LAI averaged for period of 2010-2012. 

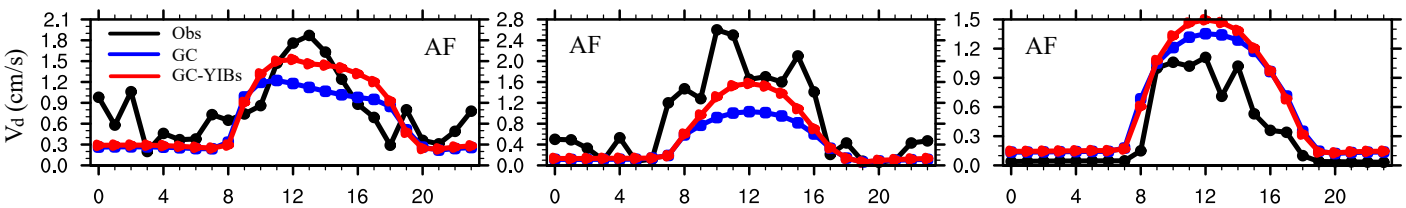

(d) Park et al. $2014\left(40.1^{\circ} \mathrm{N}, 105.3^{\circ} \mathrm{W}\right)$

(e) Finkelstein et al. $2000\left(43.6^{\circ} \mathrm{N}, 75.2^{\circ} \mathrm{W}\right)\left(\right.$ f) Hole et al. $2004\left(60.2^{\circ} \mathrm{N}, 11.4^{\circ} \mathrm{E}\right)$
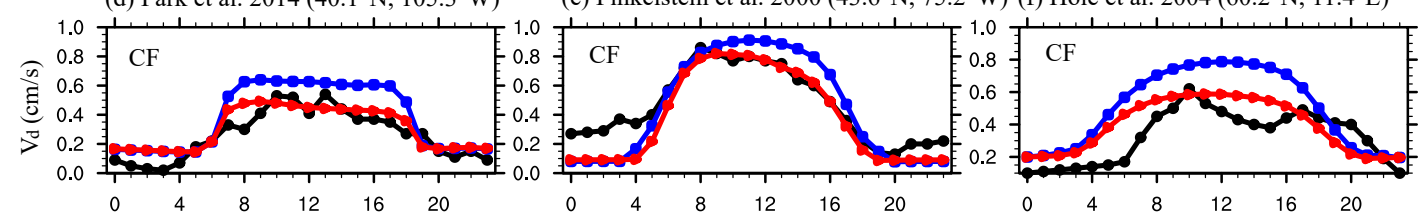

(g) Park et al. $2014\left(40.1^{\circ} \mathrm{N}, 105.3^{\circ} \mathrm{W}\right)$

(h) Mikkelsen et al. $2004\left(56.3^{\circ} \mathrm{N}\right)$

(i) $\mathrm{Wu}$ et al. $2016\left(44.2^{\circ} \mathrm{N}, 79.6^{\circ} \mathrm{W}\right)$
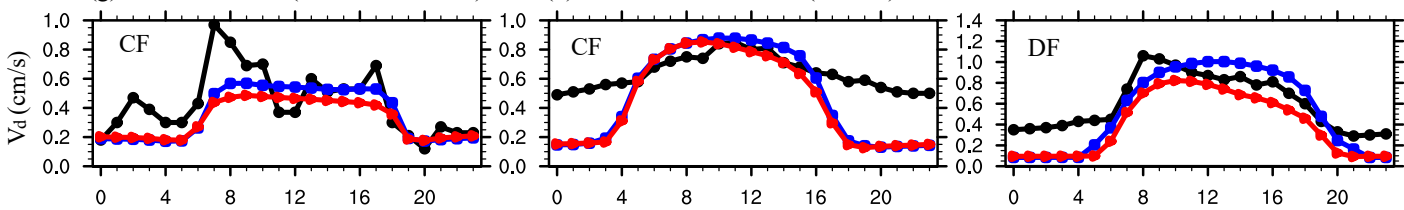

(j) Finkelstein et al. $2000\left(41.6^{\circ} \mathrm{N}, 78.8^{\circ} \mathrm{W}\right)$ (k) Munger et al. $1996\left(42.3^{\circ} \mathrm{N}, 72.1^{\circ} \mathrm{W}\right)$

(l) Padro $1996\left(44.2^{\circ} \mathrm{N}, 80.6^{\circ} \mathrm{W}\right)$
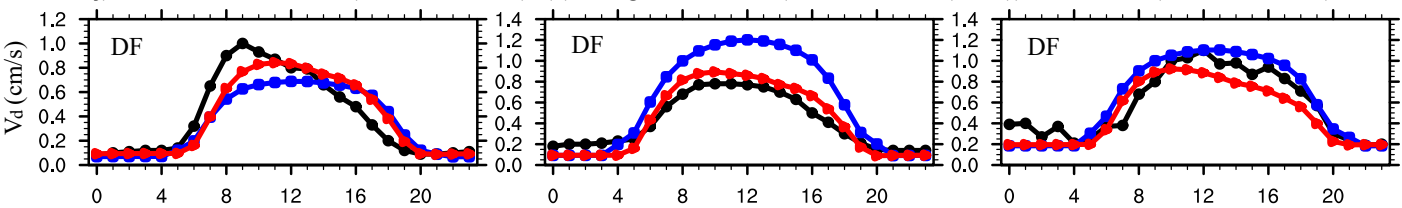

(m) Wu et al. $2011\left(42.5^{\circ} \mathrm{N}, 72.2^{\circ} \mathrm{W}\right)$

(n) Zhang et al. $2003\left(41.6^{\circ} \mathrm{N}, 78.8^{\circ} \mathrm{W}\right)$

(o) Fowler et al. $2009\left(51.2^{\circ} \mathrm{N}, 0.84^{\circ} \mathrm{W}\right)$
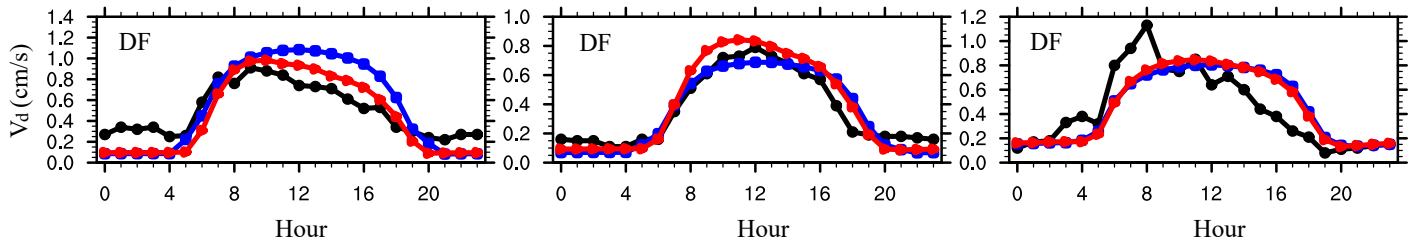

64

Figure S6 Observed and simulated diurnal cycle of $\mathrm{O}_{3}$ dry deposition velocity over Amazon (a-c), coniferous (d-h) and deciduous (i-o) forests. The black lines represent observed $\mathrm{O}_{3}$ dry deposition velocity. The blue and red lines represent simulated $\mathrm{O}_{3}$ dry deposition velocity from GC (Offline simulation) and GC-YIBs (Online_ALL simulation) models, respectively. 
(a) Low resolution of $4^{\circ} \times 5^{\circ}$

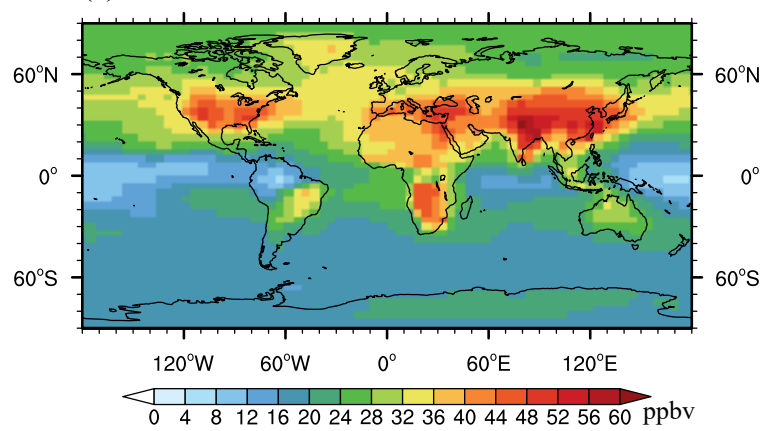

(b) High resolution of $2^{\circ} \times 2.5^{\circ}$

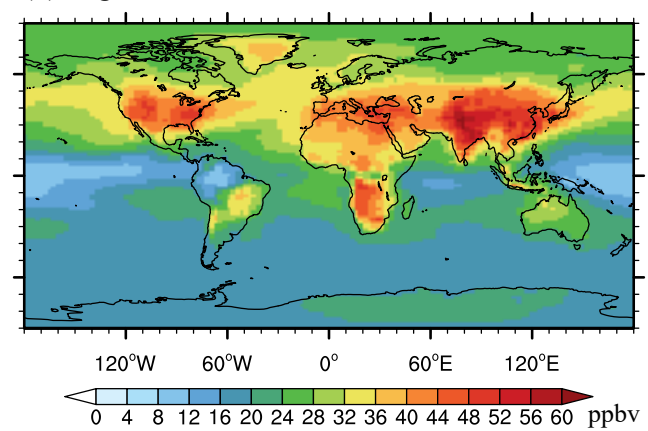

(c) Low resolution - High resolution

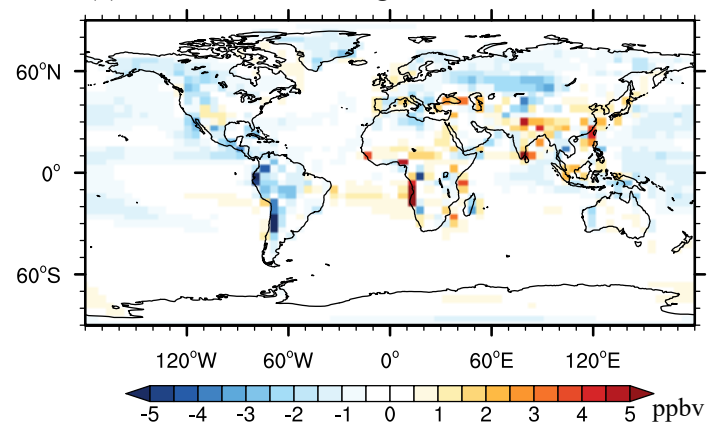

72 Figure $\mathbf{S 7}$ Comparison of $\mathrm{MDA} 8\left[\mathrm{O}_{3}\right]$ simulated with low $\left(4^{\circ} \times 5^{\circ}\right)$ (a) and relatively between low and high resolutions (a-b). 